Europhys. Lett., 51 (3), pp. 300-306 (2000)

\title{
Selection and competition of Turing patterns
}

\author{
B. PeÑA and C. PÉREz-García \\ Instituto de Física, Facultad de Ciencias, Universidad de Navarra \\ E-31080 Pamplona, Navarra, Spain
}

(received 3 December 1999; accepted in final form 31 May 2000)

PACS. 47.54.+r - Pattern selection; pattern formation.

PACS. 82.40.Bj - Oscillations, chaos, and bifurcations in homogeneous nonequilibrium reactors.

PACS. 47.20.Ky - Nonlinearity (including bifurcation theory).

\begin{abstract}
We examine the selection and competition of patterns in the Brusselator model, one of the simplest reaction-diffusion systems giving rise to Turing instabilities. Simulations of this model show a significant change in the wave number of stable patterns as the control parameter is increased. A weakly nonlinear analysis makes it possible to obtain the amplitude equations for the concentration fields near the instability threshold. Together with the linear diffusive terms, these equations also contain nonvariational spatial terms. When these terms are included, the stability diagrams and the thresholds for secondary instabilities are heavily modified with respect to the usual diffusive case. The results obtained from the numerical simulations fit very well into the calculated stability regions.
\end{abstract}

Several systems out of equilibrium exhibit pattern formation. The amplitude formalism introduced by Newell, Whitehead and Segel [1] allows spatial modulations of these patterns to be described close to a supercritical bifurcation point. Recently, several authors have discussed the necessity of including nonlinear spatial terms into generalized amplitude equations (GAE) at the leading orders for subcritical bifurcations [2,3]. The role and the weight of these terms is still under discussion. The main aim of this paper is to determine the GAE for some kind of Turing patterns arising in reaction-diffusion systems. These patterns result from a coupling between nonlinear kinetic and diffusion of reactants in which two opposed mechanisms are involved: autocatalysis (activation) and an inhibitor process [4]. In recent years, the interest in Turing patterns has been renewed with the experimental evidences of the CIMA (ChloriteIodide Malonic Acid) and CDIMA reaction (Chlorine Dioxide-Iodine Malonic Acid) [5,6]. These oscillatory reduction-oxidation reactions consist of the oxidation of $\mathrm{I}_{2}$ by $\mathrm{Cl}$ or $\mathrm{ClO}_{2}$ and the iodination of malonic acid. The difference between the diffusion coefficients of the activator $\left(\mathrm{I}^{-}\right)$and the inhibitor species $\left(\mathrm{Cl}^{-}\right.$or $\left.\mathrm{ClO}_{2}^{-}\right)$necessary to have Turing patterns is reached by using the starch as color indicator, because it decreases the diffusivity of the activator [7]. Several kinds of steady pattern have been observed: stripes, hexagons, rhombs, mixed modes, black eyes, etc. [8].

Realistic reactive schemes are so complicated that analytical results become unattainable. Therefore, we analyse the simple Brusselator model [9], which exhibits Turing patterns similar 
to those found in experiments. In terms of dimensionless variables, this model is given by two coupled reaction-diffusion equations for concentrations of the activator $X$ and the substrate $Y$ :

$$
\begin{aligned}
& \partial_{t} X=A-(B+1) X+X^{2} Y+\nabla^{2} X, \\
& \partial_{t} Y=B X-X^{2} Y+D \nabla^{2} Y,
\end{aligned}
$$

in which the parameter $D$ is proportional to the rate of diffusion coefficients $D_{Y} / D_{X}$. As usual we consider the concentration $B$ to be the control parameter.

This system presents the stationary homogeneous solution $\boldsymbol{u}_{\mathrm{s}}=\left(X_{\mathrm{s}}, Y_{\mathrm{s}}\right)=(A, B / A)$. A linear stability analysis around this solution shows that a Turing instability arises when $\eta=\sqrt{1 / D}<\left(\sqrt{1+A^{2}}-1\right) / A$ (otherwise a Hopf bifurcation takes place), at the critical values $B_{\mathrm{c}}=(1+A \eta)^{2}, k_{\mathrm{c}}=\sqrt{A \eta}[10]$. From here on, we will restrict the analysis to this case.

A multiple-scale perturbative analysis yields the well-known amplitude equations [11]. This method is based on the fact that near the instability threshold the basic state is unstable only in regard to perturbations with wave numbers close to the critical value, $k_{\mathrm{c}}$. Then, the solution can be expanded as

$$
\vec{u}=\vec{u}_{\mathrm{s}}+\sum_{j=1}^{3} \vec{u}_{0}\left(A_{j} e^{i \boldsymbol{k}_{j} \cdot \boldsymbol{r}}+\text { c.c. }\right),
$$

where $\vec{u}_{\mathrm{s}}$ represents the steady uniform state and $\vec{u}_{0}=(1,-\eta(1+A \eta) / A)^{T}$ is the eigenvector of the linearized operator. We focus the analysis on hexagonal patterns, including stripes as a particular case. The general form of the GAE for such patterns can be deduced from symmetry arguments $[2,3]$.

Up to third order in the perturbations the spatio-temporal evolution of the amplitudes $A_{j}$ is described through the equations

$$
\begin{aligned}
\tau_{0} \partial_{t} A_{1}= & \mu A_{1}+\xi_{0}^{2} \partial_{x_{1}}^{2} A_{1}-g\left|A_{1}\right|^{2} A_{1}-h\left(\left|A_{2}\right|^{2}+\left|A_{3}\right|^{2}\right) A_{1}+ \\
& +v \bar{A}_{2} \bar{A}_{3}+i \alpha_{1}\left[\bar{A}_{2} \partial_{x_{3}} \bar{A}_{3}+\bar{A}_{3} \partial_{x_{2}} \bar{A}_{2}\right]+i \alpha_{2}\left[\bar{A}_{2} \partial_{\tau_{3}} \bar{A}_{3}-\bar{A}_{3} \partial_{\tau_{2}} \bar{A}_{2}\right]
\end{aligned}
$$

where we use the standard notation for the derivatives in the parallel $\left(\partial_{x_{i}} \equiv \hat{n}_{i} \cdot \nabla\right)$ and the orthogonal $\left(\partial_{\tau_{i}} \equiv \hat{\tau}_{i} \cdot \nabla\right)$ directions to the wave vectors of the hexagonal pattern.

The form of this equation deserves some comments. First we assume that the cubic nonlinearities saturate the instability, i.e., $h, g>0$. In the Brusselator this requires that $0.564<A \eta<2.418$. Outside this range higher-order contributions must be considered [12]. Second, the spatial derivatives must be changed into $\hat{n}_{1} \cdot \nabla \rightarrow \partial_{x}-\frac{i}{2 k_{\mathrm{c}}} \partial_{y}^{2}$ for stripes. In this case $\left(v=\alpha_{i}=0\right)$ the so-called Newell-Whitehead-Segel equation [1] is recovered. Finally, the quadratic terms are assumed to be small enough, otherwise the subcritical bifurcation is ill defined. It is worth mentioning that Gunaratne et al. [13] proposed a model from which amplitude equations with nonlinear spatial terms are derived. These spatial derivatives are introduced through an operator $\square=\hat{n} \cdot \nabla-i \nabla^{2} / 2 k_{\mathrm{c}}$ which preserves the rotational invariance of the model. However, consistency of a perturbation scheme would require terms in the form $A \nabla^{2} A$ together with higher-order terms in the amplitude development. (For a detailed discussion, see ref. [14].)

The parameter $\mu=\left(B-B_{\mathrm{c}}\right) / B_{\mathrm{c}}$ stands for the supercriticallity, $\tau_{0}$ for the relaxation time and $\xi_{0}$ for the correlation length of perturbations. For the Brusselator model, the last two coefficients are given by

$$
\tau_{0}^{-1}=\frac{1+A \eta}{1-\eta^{2}}, \quad \xi_{0}^{2}=\frac{4}{(1+A \eta)^{2}} .
$$


On the other hand, the nonlinear coefficients are given in ref. [10]

$$
\begin{aligned}
& v=2 \frac{(1-A \eta)}{A(1+A \eta)}+\frac{2}{A} \mu \equiv v_{0}+v_{1} \mu \\
& g=\frac{\left(-8+38 A \eta+5 A^{2} \eta^{2}-8 A^{3} \eta^{3}\right)}{9 A^{3} \eta(1+A \eta)} \\
& h=\frac{\left(-3+5 A \eta+7 A^{2} \eta^{2}-3 A^{3} \eta^{3}\right)}{A^{3} \eta(1+A \eta)}
\end{aligned}
$$

and the spatial coefficients are [15]

$$
\alpha_{1}=\frac{4 \sqrt{A \eta}}{A(1+A \eta)^{2}}-\frac{1}{\sqrt{3}} \alpha_{2}, \quad \alpha_{2}=\frac{-2 \sqrt{3} \sqrt{A \eta} \eta(1-A \eta)}{A^{2}\left[A^{2}+\eta^{2}(1+A \eta)^{2}\right]} .
$$

The quadratic terms break the symmetry $A \rightarrow-A$ and are responsible for the subcriticality of the bifurcation. Depending on the sign of $v$, the total phase of stable hexagons is 0 or $\pi$. These solutions, labelled $H_{0}(v>0)$ and $H_{\pi}(v<0)$ in the following, correspond to a maximum or a minimum honeycomb lattice, respectively. As distinguished from other systems, the coefficient $v$ depends on the control parameter. As a result, the hexagons become stable in two separated intervals of $\mu$ (initial and re-entrant hexagons). The term with $\alpha_{1}$ corresponds to wave number dilatations, while that with $\alpha_{2}$ stabilizes stretch distortions in the pattern [3]. Coefficient $\alpha_{1}$ is always positive, much greater than $\alpha_{2}$, but of the same order as $v_{0}$.

Now we consider the stability of stationary solutions (stripes or hexagons) of eq. (3), first under spatially homogeneous perturbations (amplitude instabilities) and second under longwavelength inhomogeneities (phase instabilities).

Amplitude instabilities. - Let us consider a slight perturbation of the critical wave number $k=k_{\mathrm{c}}+q$. We begin with a striped solution of eq. (3) given by $A_{1}=S e^{i q x_{1}}, A_{2}=A_{3}=0$, where $S(\mu, q)=\sqrt{\left(\mu-\xi_{0}^{2} q^{2}\right) / g}$. A linear stability analysis around this solution is easily performed after considering disturbances in the form: $A_{1}=S\left(1+r_{1}\right) e^{i q x_{1}}, A_{2}=r_{2} e^{i q x_{2}}$ and $A_{3}=r_{3} e^{i q x_{3}}$. The stability conditions lead to the eigenvalue problem

$$
\lambda_{ \pm}(\mu, q)=(g-h) S^{2} \pm\left|v^{\prime}\right| S<0,
$$

where $v^{\prime}=v+2 \alpha_{1} q$. After introducing the expressions of the coefficients $g, h$ and $v$ into this equation, it is easy to derive that stripes are unstable for any value of the control parameter within $0<A \eta<0.879$. On the other hand, if $0.879<A \eta<2.418$, the equations $\lambda_{ \pm}=0$ determine the limits of stripe stability, $\mu_{S \pm}(q)$ (see fig. 1).

Now, let us consider disturbances in the form $A_{i}=H e^{i q x_{i}}$ around the steady hexagonal solution of eq. (3), $H(\mu, q)=\left(v^{\prime}+\sqrt{v^{\prime 2}+4(g+2 h)\left(\mu-\xi_{0}^{2} q^{2}\right)}\right) / 2(g+2 h)$. The stability conditions read as

$$
\begin{aligned}
w(\mu, q) & =2(g+2 h) H^{2}-\left|v^{\prime}\right| H>0, \\
u(\mu, q) & =(g-h) H^{2}+\left|v^{\prime}\right| H>0 .
\end{aligned}
$$

(Note that we use $\left|v^{\prime}\right|$ to describe both $H_{0}$ and $H_{\pi}$ hexagons.) The condition $w(\mu, q)=0$ gives a marginal curve $\mu_{\mathrm{M}}(q)$, above which hexagons can appear. The second inequality is satisfied whenever $0<A \eta<0.953$. Within this range the $H_{0}$-hexagons are the only stable solution above $\mu_{\mathrm{M}}$. However, for $0.953<A \eta<2.418$, the condition (6) is not satisfied (unstable hexagons) inside $\mu_{\mathrm{H}-}<\mu<\mu_{\mathrm{H}+}$ given by $u(\mu, q)=0$. The last case corresponds to reentrant hexagons. For $A \eta<1\left(v_{0} v_{1}>0\right)$ the total phase of hexagons is unchanged $\left(H_{0}\right)$, 


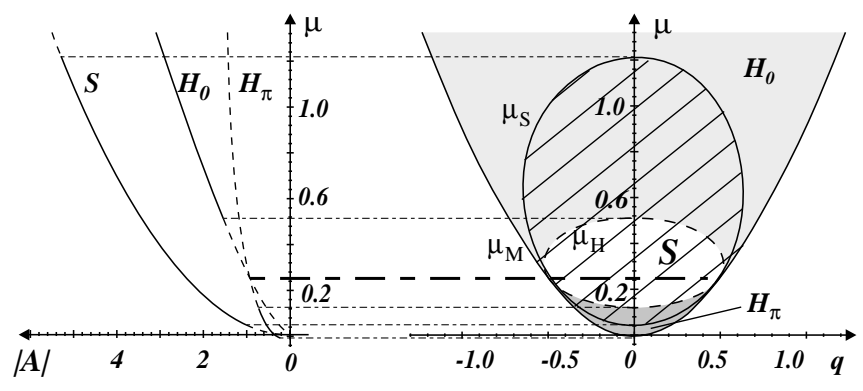

Fig. 1 - Stability diagrams for $A=4.5$ and $A \eta=1.59$ neglecting the nonlinear spatial terms (see the text). Dark regions correspond to stable hexagons while striped domains indicate stable stripes. Notice that the curves are symmetrical with respect to the vertical axis. The left diagram shows the amplitude of stable solutions with $k=k_{\mathrm{c}}$ as a function of $\mu$.

whereas for $A \eta>1$ the parameter $v^{\prime}$ changes its sign when $\mu$ increases; then the total phase is changed from 0 to $\pi$. An example of this case neglecting the $\alpha_{i}$ terms is shown in fig. 1 . Hexagons are stable in the dark regions, and the stripes in the striped area. On the left side of fig. 1 we represent the usual bifurcation diagram $(q=0)$.

Phase instabilities. - The stripes are disturbed also in the phase $\phi: A=S(1+r+i \phi) e^{i q x}$. Assuming that the amplitude perturbation $r$ is enslaved by the phase, one arrives at the usual phase equation [11]

$$
\partial_{t} \phi=D_{\|} \partial_{x}^{2} \phi+D_{\perp} \partial_{y}^{2} \phi
$$

where $D_{\|}=\left(\mu-3 \xi_{0}^{2} q^{2}\right) /\left(\mu-\xi_{0}^{2} q^{2}\right)$ and $D_{\perp}=\xi_{0} q / k_{\mathrm{c}}$ are the diffusion coefficients. The Eckhaus instability takes place when $D_{\|}<0$, whereas the zig-zag instability occurs if $D_{\perp}<0$, i.e., for wavelengths greater than the critical one.

Following similar steps, for a slightly distorted hexagonal pattern given by $A_{i}=H\left(1+r_{i}+\right.$ $\left.i \phi_{i}\right) e^{i q x_{i}}, i=1,2,3$, the corresponding phase equation is obtained. As before, the amplitudes $r_{i}$ and the total phase $\Phi=\phi_{1}+\phi_{2}+\phi_{3}$ are enslaved modes that can be eliminated adiabatically [16]. Therefore, only two of the three phases of a hexagonal structure are independent. A center manifold reduction to the phase modes $\phi_{x}=-\left(\phi_{2}+\phi_{2}\right)$ and $\phi_{y}=\left(\phi_{2}-\phi_{3}\right) / \sqrt{3}$ leads to the general form of the hexagonal phase equation [17]:

$$
\partial_{T} \vec{\phi}=D_{\mathrm{t}} \nabla^{2} \vec{\phi}+\left(D_{\mathrm{l}}-D_{\mathrm{t}}\right) \nabla(\nabla \cdot \vec{\phi}),
$$

where the coefficients are given by the expressions

$$
\begin{aligned}
D_{\mathrm{t}}= & \frac{1}{4}-\frac{\xi_{0}^{2} q^{2}}{2 u}+\frac{H^{2}}{8 u}\left(\alpha_{1}-\sqrt{3} \alpha_{2}\right)^{2} \\
D_{\mathrm{l}}= & \frac{3}{4}-\frac{\xi_{0}^{2} q^{2}(4 u+w)}{2 u w}+\frac{H^{2}}{8 u}\left(\alpha_{1}-\sqrt{3} \alpha_{2}\right)^{2}- \\
& -\frac{\alpha_{1} H^{2}}{w}\left(\alpha_{1}+\sqrt{3} \alpha_{2}\right)+\frac{\xi_{0} q H}{w}\left(3 \alpha_{1}+\sqrt{3} \alpha_{2}\right)
\end{aligned}
$$

(details can be found in ref. [3]). The rhs of the phase equation for hexagons is similar to the wave equation of an isotropic elastic solid [18], so we denote the diffusion coefficients with subscripts 1 and $\mathrm{t}\left(D_{\mathrm{l}}\right.$ and $\left.D_{\mathrm{t}}\right)$ by analogy with the speed of longitudinal and transversal 


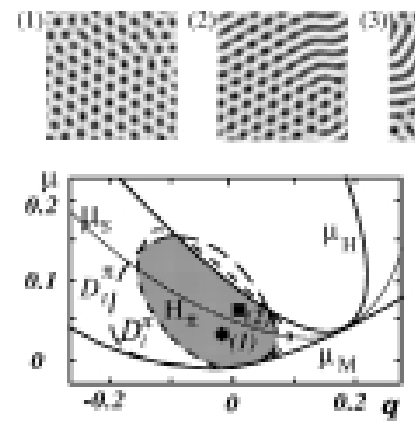

(a)

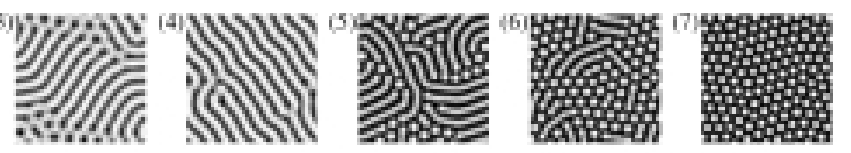

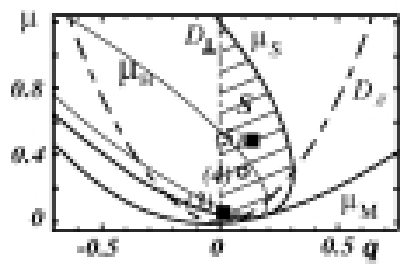

(b)

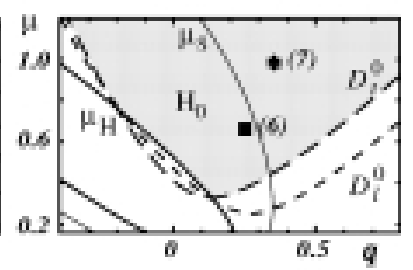

(c)

Fig. 2 - Amplitude (solid lines) and phase instabilities (dashed curves) for $A \eta=1.59$. (a) Initial hexagons $\left(H_{\pi}\right)$ and their coexistence with stripes. (b) Stability of stripes and spatially mixed patterns. (c) Re-entrant $\left(H_{0}\right)$ hexagons. The simulated patterns (grid: $\left.128 \times 128\right)$ have been obtained for the values: (1) $\mu=0.04$, (2) $\mu=0.06$, (3) $\mu=0.07$, (4) $\mu=0.09$, (5) $\mu=0.30$, (6) $\mu=0.66$ and (7) $\mu=0.98$.

sound waves. Following with this analogy the phase field can be split into two normal modes $\vec{\phi}=\vec{\phi}_{1}+\vec{\phi}_{\mathrm{t}}$, that obey the relationships $\nabla \times \vec{\phi}_{\mathrm{l}}=0, \nabla \cdot \vec{\phi}_{\mathrm{t}}=0$. Therefore, the phase equation is equivalent to the diffusion equations: $\partial_{\mathrm{t}} \vec{\phi}_{1, \mathrm{t}}=D_{\mathrm{l}, \mathrm{t}} \nabla^{2} \vec{\phi}_{\mathrm{l}, \mathrm{t}}$. A given pattern remains stable under phase perturbations whenever $D_{1, \mathrm{t}}>0$. The two normal modes $\vec{\phi}_{\mathrm{l}}$ and $\vec{\phi}_{\mathrm{t}}$ correspond to rectangular and rhomboidal perturbations, respectively.

In particular we show the case $A \eta=1.59$ with some detail in fig. 2 . Solid lines denote amplitude instabilities and dashed curves correspond to phase instabilities. As occurs in the amplitude stability diagrams, the phase curves are asymmetrical when the nonlinear spatial terms are included. In fig. $2 \mathrm{a}, H_{\pi}$-hexagons are stable in the dark region, limited by the lines labelled $D_{\mathrm{t}, 1}^{\pi}$, that denote the curves $D_{\mathrm{t}, 1}^{\pi}\left(H_{\pi}\right)=0$. Above the curve $\mu_{S-}$, the $H_{\pi}$-hexagons coexist with stripes (patterns 2 and 3 ). Figure $2 \mathrm{~b}$ corresponds to intermediate values of the control parameter. The dashed region corresponds to stable stripes. Figure 2c contains the stability region of $H_{0}$-hexagons. In this case, $D_{\mathrm{t}, 1}^{0}$ stands for the curves $D_{\mathrm{t}, 1}^{0}\left(H_{0}\right)=0$.

Points obtained from numerical simulations of the Brusselator and the corresponding pat-

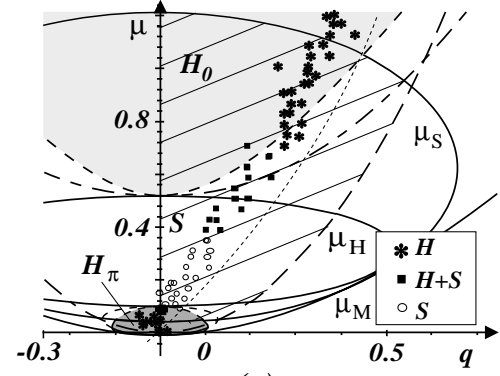

(a)

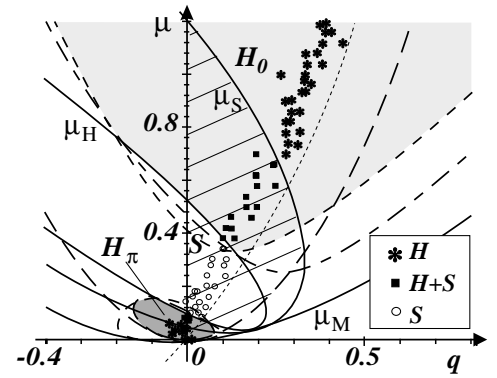

(b)

Fig. 3 - Amplitude (solid lines) and phase instabilities (dashed curves) for $A \eta=1.59$ (a) neglecting the terms in $\alpha_{i}$ and (b) including the corresponding values $\alpha_{1}=0.21, \alpha_{2}=-2.7 \cdot 10^{-3}$. - - Eckhaus instability, - - curve $D_{1}=0$ and $--D_{\mathrm{t}}=0$. The points correspond to values obtained by simulations of the Brusselator. The dotted line indicates the maximum linear growth. 
terns have also been included in this figure. Simulations were performed by using the semiimplicit method reported in ref. [19], starting from random initial conditions. The patterns labelled (1), (4) and (7) are located in stability regions in which only one kind of symmetry is possible $\left(H_{\pi}\right.$-hexagons, stripes, and $H_{0}$-hexagons, respectively). On the other hand, points (2) and (3) fit within the range of coexistence of $H_{\pi}$-hexagons and stripes, and points (5) and (6) are located in the region of competition between stripes and $H_{0}$-hexagons. These patterns show complete agreement between the model and the stability regions obtained from the GAE, and are quite similar to those found in experiments.

For the sake of comparison, we show in fig. 3a the amplitude stability diagram (fig. 1) with the phase stability curves neglecting the terms in $\alpha_{i}$. We also include the curve of maximum linear growth (dotted line) and points obtained from simulations. Mixtures of states $(H+S)$ stand in a region where only stripes are predicted to be stable, and pure $H_{0}$-hexagons appear in simulations, while theory predicts that a mixed $H+S$ mode can be stable.

When nonvariational terms are taken into account, the results shown in fig. $3 \mathrm{~b}$ are obtained (after gathering together the diagrams of fig. 2). The wave number in simulations increases with the supercriticallity and the corresponding points remain not far from the maximum linear growth curve. The points fit remarkably well into the stability regions of this asymmetrical diagram (fig. 3b). This comparison shows that the spatial nonlinear terms are crucial to explain the onset of instabilities and the nonlinear pattern selection in the Brusselator.

We present a detailed theoretical analysis of spatial effects in Turing patterns in the Brusselator. The generalized amplitude equations are derived taking consistently into account spatial modulations. For hexagonal patterns, these equations include spatial nonvariational terms $[2,3]$, which describe distorted hexagons and permit wave number variations from the critical value. From the amplitude equations, the stability of patterns towards uniform and inhomogenous perturbations is determined. Then, the phase equations are derived and the phase stability diagrams are obtained. The spatial nonlinear terms yield asymmetrical contributions, changing the thresholds and the critical wave numbers of instabilities. Numerical simulations of the Brusselator thus fit quite well into the stability regions calculated with those terms (see fig. 3b), then, providing support for these generalized amplitude equations.

\section{$* * *$}

We wish to thank Dr. B. Echebarria and Prof. H. Mancini (Pamplona) for helpful comments and discussions. This work was partly supported by a DGICYT grant PB98-0208 (Spanish Goverment), Universidad de Navarra (PIUNA) and Integrated Action Italy-Spain HI97-30. BP acknowledges the financial support of the Association "Amigos de la Universidad de Navarra" and "Caja de Ahorros de Pamplona".

\section{REFERENCES}

[1] Newell A. C. and Whitehead J. A., J. Fluid Mech., 38 (1969) 203.

[2] Brand H., Progr. Theor. Phys. Suppl., 99 (1989) 442; Kuznetsov E. A., Nepomnyashchy A. A. and Pismen L. M., Phys. Lett. A, 205 (1995) 261.

[3] Echebarria B. and Pérez-García C., Europhys. Lett., 43 (1998) 35.

[4] Turing A. M., Philos. Trans. R. Soc. London B, 327 (1952) 37.

[5] Castets V., Dulos E., Boissonade J. and De Kepper P., Phys. Rev. Lett., 64 (1990) 2953.

[6] Ouyang Q. and Swinney H. L., Nature, 352 (1991) 610.

[7] Lengyel I. and Epstein I. R., Science, 251 (1991) 650; Noszticzius Z., Ouyang Q., McCormick W. D. and Swinney H. L., J. Phys. Chem., 96 (1992) 6302. 
[8] Kapral S. and Showalter K., Chemical Waves and Patterns (Klüwer, Amsterdam) 1995.

[9] Prigogine I. and Lefever R., J. Chem. Phys., 48 (1968) 1695.

[10] De Wit A., Ph. D. Thesis, Université Libre de Bruxelles (1993).

[11] Manneville P., Dissipative Structures and Weak Turbulence (Academic Press, San Diego) 1990.

[12] Hilali M., Métens S., Borckmans P. and Dewel G., Phys. Rev. E, 51 (1995) 2046.

[13] Gunaratne G. H., Ouyang Q. and Swinney H. L., Phys. Rev. E, 50 (1994) 2802.

[14] See the discussion in Nepomnyashchy A. A. and Pismen L. M., Phys. Rev. Lett., 72 (1994) 944, and accompanying comment Gunaratne G. H., Phys. Rev. Lett., 72 (1994) 945.

[15] Peña B. and PÉrez-García C., in preparation.

[16] Hoyle R., Appl. Math. Lett., 9 (1995) 81.

[17] Lauzeral J., Mètens S. and Walgraef D., Europhys. Lett., 24 (1993) 707.

[18] Landau L. D. and Lifshitz E. M., Teoría de la elasticidad (Reverté, Barcelona-Buenos AiresMéxico) 1969.

[19] Dufiet V. and Boissonade J., Physica A, 188 (1992) 158. 